\title{
Labour epidural analgesia and anti-infectious management of the neonate: a meta-analysis*
}

\author{
Michael Heesen ${ }^{1, * *}$, Sven Klöhr ${ }^{1}$, Rolf Rossaint ${ }^{2}$, \\ Sebastian Straube ${ }^{3}$ and Marc Van de Velde ${ }^{4}$ \\ ${ }^{1}$ Department of Anesthesia, Klinikum Bamberg, Bamberg, \\ Germany \\ ${ }^{2}$ Department of Anesthesia, Universitätsklinkum Aachen, \\ Aachen, Germany \\ ${ }^{3}$ Department of Occupational, Social and Environmental \\ Medicine, University Medical Center Göttingen, \\ Göttingen, Germany \\ ${ }^{4}$ Department of Anesthesia, Universitair Zieckenhuis \\ Leuven, Leuven, Belgium
}

\begin{abstract}
Background: A known side effect of labour epidural analgesia (EDA) is maternal fever. It is unclear whether this has effects on the anti-infectious management of the neonate.

Methods: A systematic literature search and a hand search of abstract publications were conducted. Studies reporting sepsis evaluation or antibiotic treatment were further assessed. For meta-analysis, risk ratio (RR) and $95 \%$ confidence interval (CI) were calculated using the random effects model.

Results: Five relevant articles reporting on 4667 parturients were identified; three were observational studies and two were randomised controlled trials (RCT). The RR for sepsis workup of all studies analysed together was 2.58 (95\% CI, 1.06-6.27, $\mathrm{P}=0.04)$. The RR for antibiotic treatment of the
\end{abstract}

\footnotetext{
*Contribution to authorship: All authors made substantial contributions to the conception and design of the study, as well as the acquisition, analysis, and interpretation of data. M.H. designed and conducted the study, analysed the data, and wrote the manuscript. S.K. analysed the data and wrote the manuscript. R. Rossaint designed the study and wrote the manuscript. S.S. wrote the manuscript. M.V.V. designed and conducted the study, and wrote the manuscript. All the authors approved the final version of the manuscript. This review was done at the Department of Anesthesia, Klinikum Bamberg, Bamberg, Germany.

**Corresponding author:

Michael Heesen, MD

Department of Anesthesia

Klinikum Bamberg

Bugerstrasse 80

96049 Bamberg

Tel.: +49-951-503-13001

Fax: +49-951-503-13009

E-mail: michael.heesen@sozialstiftung-bamberg.de
}

neonate was 2.76 (95\% CI, 1.20-6.31, $\mathrm{P}=0.02)$. When considering the RCTs alone, the RRs for sepsis evaluation and antimicrobial treatment were still significantly elevated.

Discussion: Our data suggest that EDA-related maternal hyperthermia results in an increased likelihood of sepsis workup and antibiotic treatment of the infant. A crucial question is whether EDA-related maternal hyperthermia is truly infectious. If not, administration of antibiotics would not be justified and may be dangerous.

Keywords: Antibiotics; epidural anesthesia; labour analgesia; neonatal sepsis; obstetrics.

\section{Introduction}

Epidural analgesia (EDA) provides reliable pain relief in labouring women but is associated with maternal fever [7]. As demonstrated in a meta-analysis of randomised controlled trials (RCTs), the risk is increased more than two-fold [1]. Infection is difficult to diagnose during labour because traditional markers of infection including leucocyte count [10], C-reactive protein [17], or procalcitonin [12] tend to be elevated and are not useful. Abdominal tenderness may be abolished by EDA, and transvaginal uterine cultures may be contaminated with physiologic vaginal germs. Therefore, maternal fever may flag infants at an increased risk of infection. We asked whether EDA is associated with rates of sepsis workup and antibiotic treatment in the neonate. A systematic literature search followed by a meta-analysis of the available evidence was performed.

\section{Methods}

A systematic search in PubMed and Embase was done with the following search words (MeSH and free text): "epidural or peridural analgesia" or "anesthesia and labour and antibiotics or antimicrobial therapy" or "sepsis evaluation or sepsis work up" or "sepsis workup". Additionally, a hand-search was performed of abstract publications of the annual congresses of the society of obstetrics and perinatology as well as of the American and European societies of anaesthesiology.

To capture all relevant evidence, any study comparing parturients who received labour EDA with a control group receiving no EDA was eligible for inclusion regardless of study type (blinded or nonblinded) and publication type (full paper or abstract publication). The neonatal outcome parameters antibiotic administration, diagnosis of sepsis, and sepsis workup were assessed. 
The random effects model was used for the meta-analyses of these dichotomous parameters. The pooled risk ratio (RR) and $95 \%$ confidence interval $(95 \% \mathrm{CI})$ were calculated. Review Manager (RevMan) version 5.0 (The Nordic Cochrane Centre, The Cochrane Collaboration, Copenhagen, 2008) was used. We performed a sensitivity analysis by comparing the results of observational studies and RCTs.

\section{Results}

Our literature search identified 48 articles, of which five studies $[2,9,11,13,15]$ provided relevant data and were used for further analysis (Figure 1). Data of 4667 parturients were reported in the five studies. Three studies $[9,11,13]$ including 3838 patients were observational and two studies $[2,15]$ of 829 parturients were RCTs. Table 1 contains the study details. The paper by Lieberman et al. [13] was a secondary analysis of an active labour management trial that was previously published [6]. Neither the paper by Lieberman et al. nor the previous report detailed the analgetic procedure of epidural and non-epidural groups.
Fever was defined as temperature $>38^{\circ} \mathrm{C}$ in three studies [2, $11,13], \geq 38^{\circ} \mathrm{C}$ in the study by Philip et al. [15], and $>37.8^{\circ} \mathrm{C}$ in the report by Gonen et al. [9].

The RR for sepsis workup of all studies together was 2.58 (95\% CI, 1.06-6.27; P=0.04; Figure 2A). Eliminating the RCT [15] from the analysis, the RR was 2.50 (95\% CI, 0.75$8.38)$, which was no longer significant $(\mathrm{P}=0.14)$. The $\mathrm{RR}$ of the RCT [15] alone was 1.51 (95\% CI, 1.12-2.04).

The RR for antibiotic treatment of the neonate was 2.76 (95\% CI, 1.20-6.31, P=0.02) when considering all studies (Figure 2B). The RR of the observational studies alone was 5.30 (95\% CI, 3.42-8.22, $\mathrm{P}=0.00001)$ and was higher than the RR of the two RCTs $[2,15](1.61 ; 95 \%$ CI, 1.14-2.27, $\mathrm{P}=0.006$ ).

\section{Discussion}

To the best of our knowledge, this is the first systematic review and meta-analysis addressing the question whether labour EDA affects anti-infectious management of the infant.

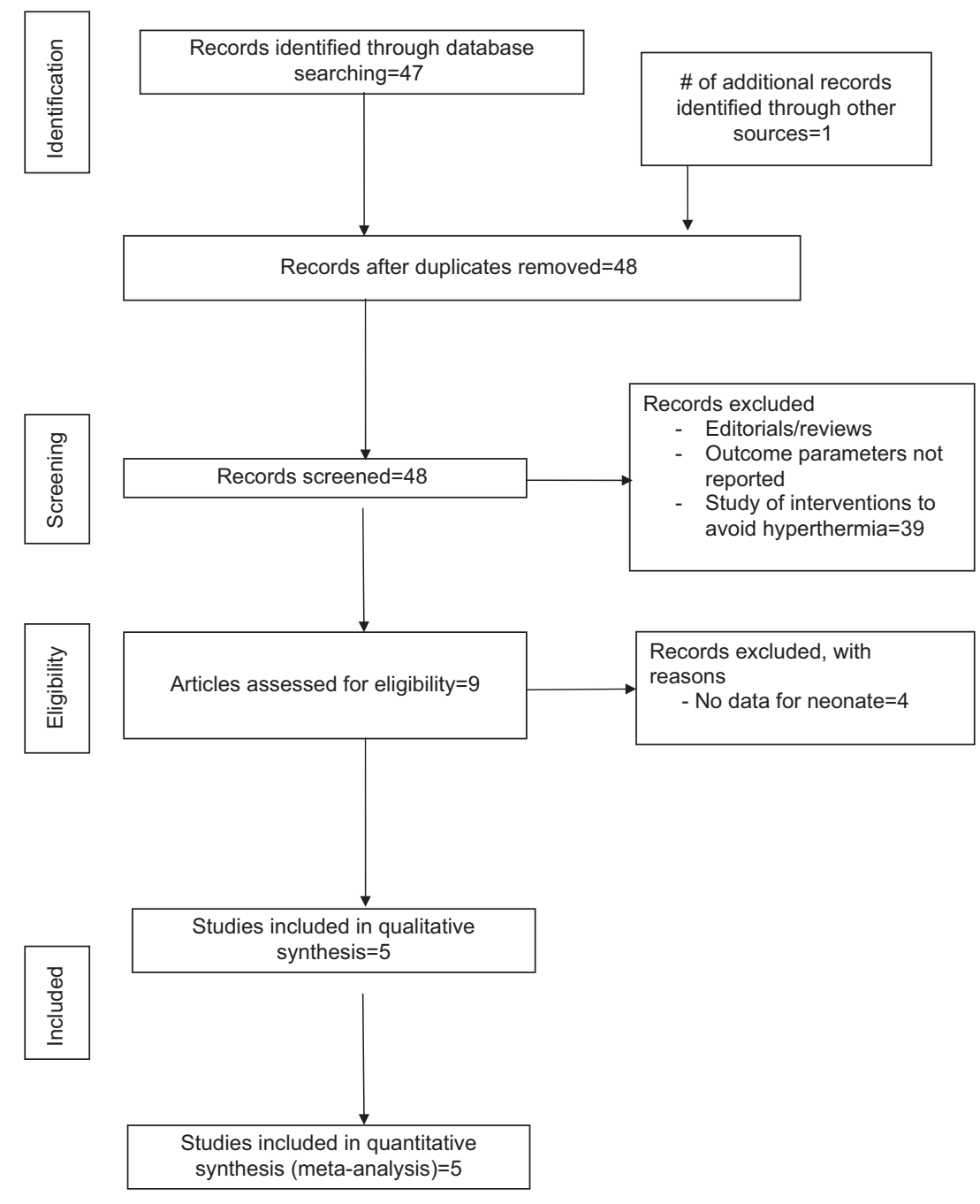

Figure 1 Flow diagram. 


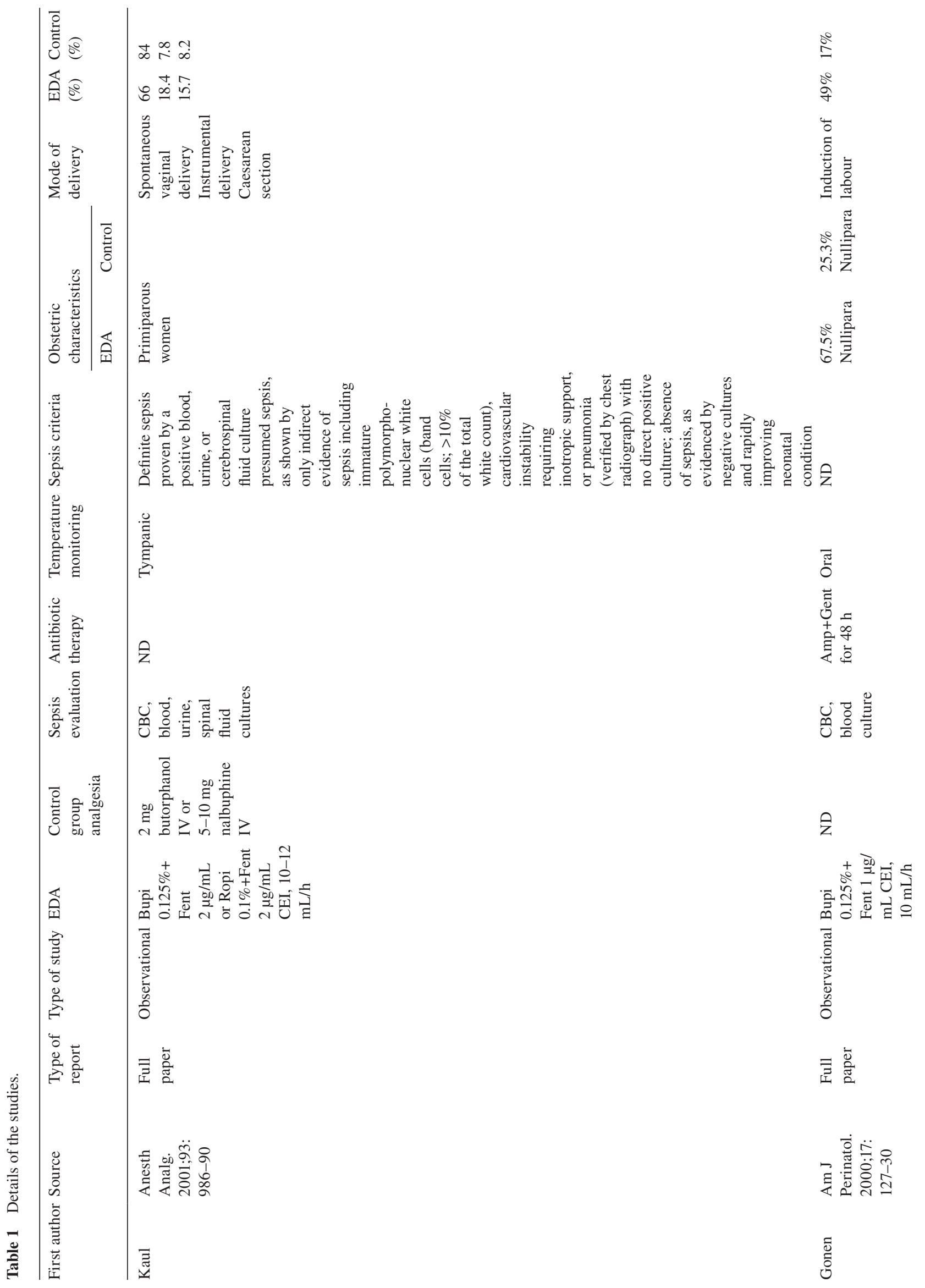




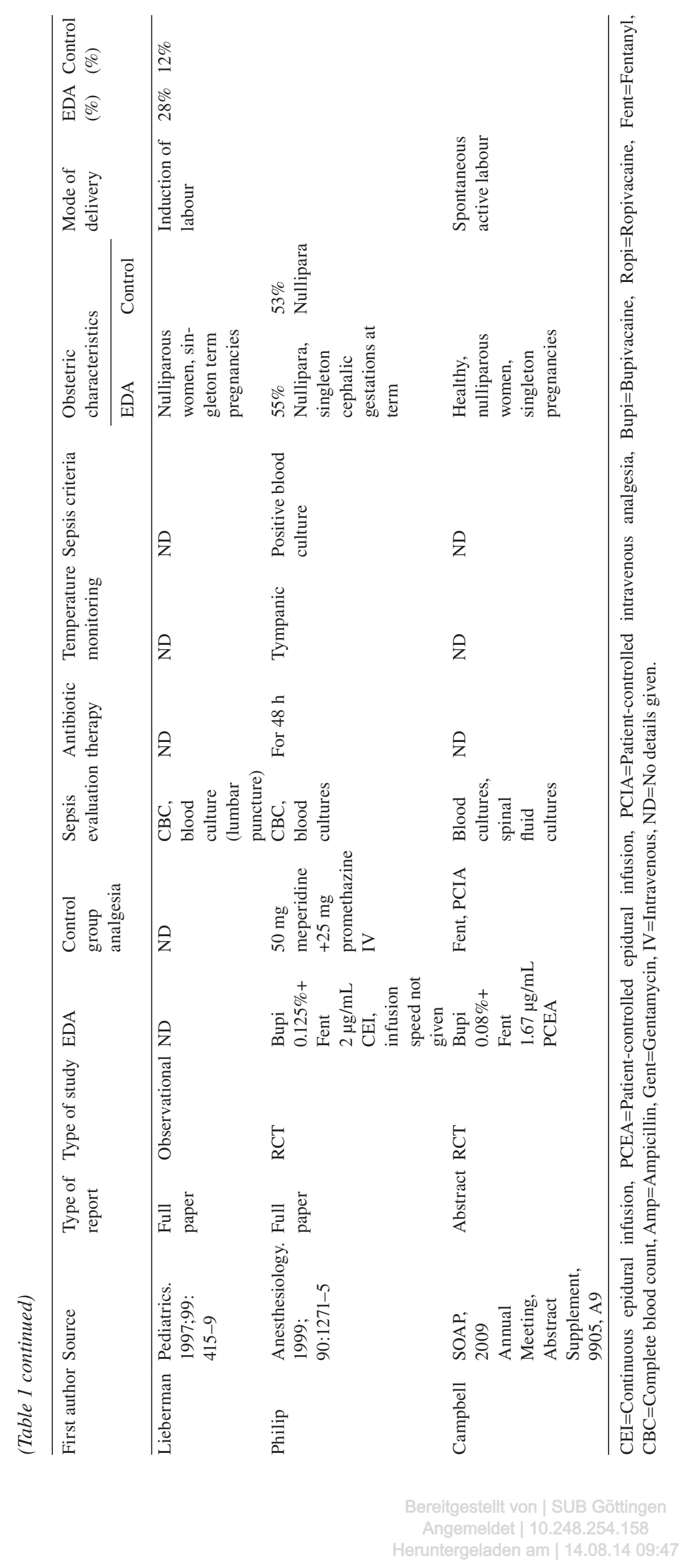


A

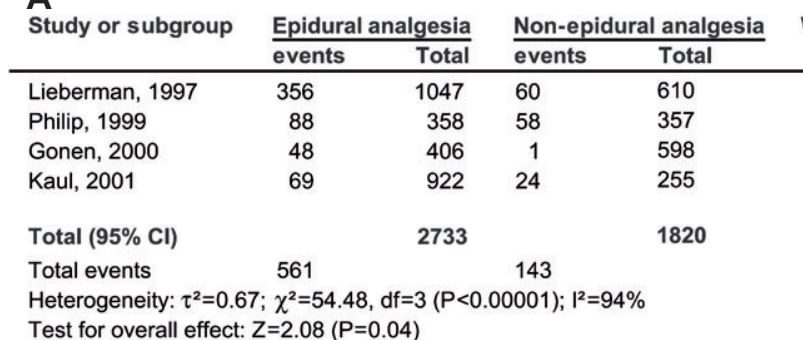

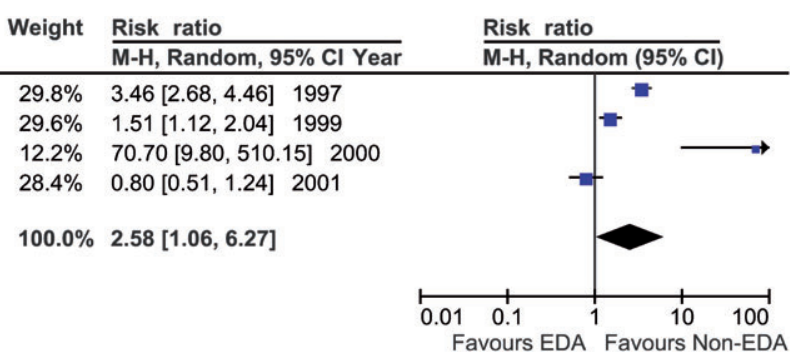

B

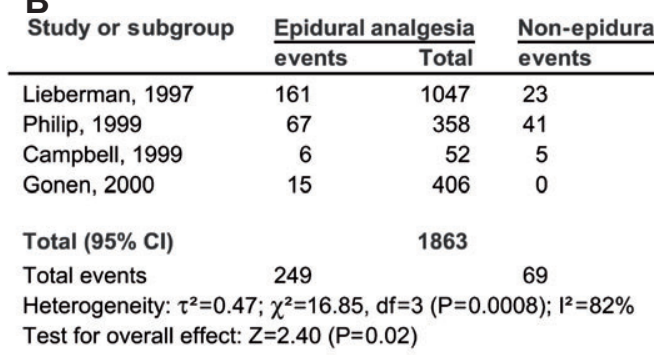

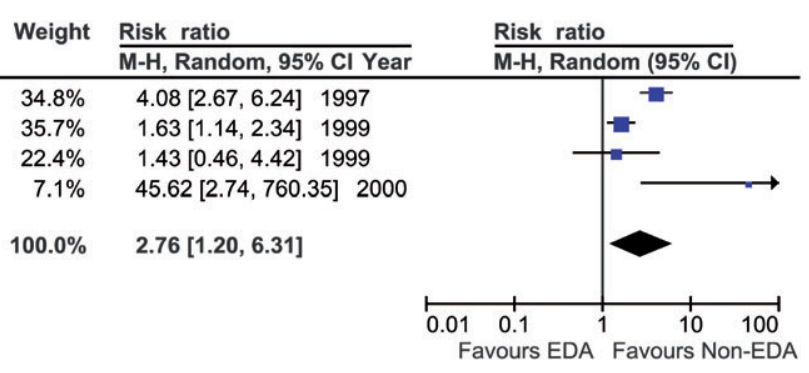

Figure 2 (A) Sepsis workup of neonates of mothers receiving EDA vs. non-EDA. (B) Antibiotic treatment of neonates of mothers receiving EDA vs. non-EDA.

Although the number of studies reporting on our outcome parameters of interest is low, 4553 parturients are a sufficiently high number of subjects to ensure a reliable result. Labour EDA is associated with maternal fever [1], and this may lead to the diagnosis of infection. We found that the RR for antibiotic treatment of the neonate was significantly elevated. This was true for an analysis of all studies, observational studies, and the RCT together. The RCTs alone also found a significantly increased RR.

Moreover, the infants of mothers treated with EDA had an increased RR to be subjected to a sepsis workup. This finding is significant for the analysis of all studies together as well as the data provided in the RCT.

It is still a matter of debate whether EDA-associated fever is infectious or not. Dashe et al. [4] concluded that fever in parturients receiving EDA is a consequence of true infection; by contrast, others $[8,16]$ attributed maternal hyperthermia to a non-infectious inflammatory state. In observational studies, women receive EDA at their request and these patients might be those who have risk factors for fever, including prolonged labour and premature rupture of membrane. By contrast, an $\mathrm{RCT}$ randomly assigning parturients to the EDA or the intravenous analgesia group reduces this risk of allocation bias. Our results appear robust because the analysis of all studies together as well as the data of the RCTs alone unambiguously reveal an increased likelihood of sepsis workup and antibiotic treatment in the infant.

The studies included in our analysis date from 1997 to 2000 and thus are more than 10 years old. One might ask if today's management would be different. Maternal fever is still considered a cornerstone for the diagnosis of chorioamnionitis [5]. According to the Centers for Disease Control and Prevention guidelines [3], chorioamnionitis is diagnosed clinically and symptoms may be unspecific. If this diagnosis is made, the neonate should, according to the guidelines, be treated with antibiotics. Therefore, it is very likely that, nowadays, infants of mothers with hyperthermia during labour EDA would also receive antibiotics. In clinical practise, maternal fever alone, i.e., without the presumed diagnosis of chorioamnionitis, may also prompt the treatment of the infant with antibiotics.

The diagnosis of sepsis was based on clinical criteria that were not given in detail in four of the five studies included into our review. However, the lack of clear criteria to be used for diagnosing sepsis may reflect daily clinical practise. Also, whether to administer antibiotics to the neonate is a clinical decision and may vary between institutions and between reports.

Antibiotic treatment early in life may have long-lasting negative effects. Asthma incidence is slightly elevated in children exposed to antibiotics during pregnancy or the first year of life [14].

Our analysis clearly showed that EDA of the mother leads to an increased likelihood of sepsis workup and antibiotic treatment of the infant. A crucial question in this context is whether fever secondary to EDA is of infectious origin or not. If not, antibiotic treatment would not be justified and would unnecessarily expose the infant to side effects.

\section{References}

[1] Anim-Somuah M, Smyth RMD, Howell CJ. Epidural versus non-epidural or no analgesia in labour. Cochrane Database Syst Rev. 2005;(4):CD000331.

[2] Campbell D, Halpern St, Muir H, Breen T, Blanchard W. Maternal intrapartum fever and neonatal sepsis. Society for Obstetric Anesthesia and Perinatology, 2009 Annual Meeting, Abstract Supplement. 2009;9905:A9. 
[3] Centers for Disease Control and Prevention. Prevention of perinatal group B streptococcal disease. MMWR. 2010;59:1-32.

[4] Dashe JS, Rogers BB, McIntire DD, Leveno KJ. Epidural analgesia and intrapartum fever: placental findings. Obstet Gynecol. 1999;93:341-4.

[5] Fishman SG, Gelber SE. Evidence for the clinical management of chorioamnionitis. Semin Fetal Neonatal Med. 2012;17:46-50.

[6] Frigoletto FD Jr, Lieberman E, Lang JM, Cohen A, Barss V, Ringer S, et al. A clinical trial of active management of labor. $\mathrm{N}$ Engl J Med. 1995;333:745-50.

[7] Fusi L, Steer PJ, Maresh MJ, Beard RW. Maternal pyrexia associated with the use of epidural analgesia in labour. Lancet. 1989;I:1250-2.

[8] Goetzl L, Evans T, Rivers J, Suresh MS, Lieberman E. Elevated maternal and fetal serum interleukin-6 levels are associated with epidural fever. Am J Obstet Gynecol. 2002;187:834-8.

[9] Gonen R, Korobochka R, Degani S, Gaitini L. Association between epidural analgesia and intrapartum fever. Am J Perinatol. 2000;17:127-30.

[10] Griffin JF, Beck I. Changes in maternal peripheral leucocytes around delivery. Br J Obstet Gynaecol. 1980;87:402-7.

[11] Kaul B, Vallejo M, Ramanathan S, Mandell G. Epidural labor analgesia and neonatal sepsis evaluation rate: a quality improvement study. Anesth Analg. 2001;93:986-90.

[12] Kordek A, Torbé A, Czajka R. Maternal venous procalcitonin levels do not correlate with umbilical cord blood and venous blood concentrations in the neonate. J Perinat Med. 2006;34:462-5.

[13] Lieberman E, Lang JM, Frigoletto F Jr, Richardson DK, Ringer SA, Cohen A. Epidural analgesia, intrapartum fever, and neonatal sepsis evaluation. Pediatrics. 1997;99:415-9.

[14] Murk W, Risnes KR, Bracken MB. Prenatal or early-life exposure to antibiotics and risk of childhood asthma: a systematic review. Pediatrics. 2011;127:1125-38.

[15] Philip J, Alexander JM, Sharma SK, Leveno KJ, McIntire DD, Wiley J. Epidural analgesia during labor and maternal fever. Anesthesiology. 1999;90:1271-5.

[16] Riley LE, Celi AC, Onderdonk AB, Roberts DJ, Johnson LC, Tsen LC, et al. Association of epidural-related fever and non-infectious inflammation in term labor. Obstet Gynecol. 2011;117:588-95.

[17] Wiwanitkit V. Maternal C-reactive protein for detection of chorioamnionitis: an appraisal. Infect Dis Obstet Gynecol. 2005;13:179-81.

The authors stated that there are no conflicts of interest regarding the publication of this article and that all approved the final manuscript. Funding came from departmental funds.

Received April 3, 2012. Revised May 10, 2012. Accepted May 29, 2012. Previously published online June 24, 2012. 